Error estimation of measuring total interaction coefficients of turbid media using collimated light transmission

This content has been downloaded from IOPscience. Please scroll down to see the full text. 1994 Phys. Med. Biol. 392349

(http://iopscience.iop.org/0031-9155/39/12/015)

View the table of contents for this issue, or go to the journal homepage for more

Download details:

IP Address: 131.215.70.231

This content was downloaded on 23/07/2016 at 00:33

Please note that terms and conditions apply. 


\title{
Error estimation of measuring total interaction coefficients of turbid media using collimated light transmission
}

\author{
Lihong Wang and Steven L Jacques \\ Laser Biology Research Laboratory, Box 17, The University of Texas M D Anderson Cancer \\ Center, 1515 Holcombe Blvd, Houston, TX 77030, USA
}

Received 7 April 1994

\begin{abstract}
The error of measuring the total interaction coefficients of turbid media using collimated light transmission was estimated with an analytical expression, which was verified with accurate Monte Carlo simulations. The expression is based on the Henyey-Greenstein phase function of scattering and the probabilities of non-scattered and singly scattered photons transmitted through a tissue slab with a unit anisotropy factor.
\end{abstract}

\section{Introduction}

Lasers are increasingly being used in the medical sciences as tools for both diagnosis and therapy (Shapshay 1989, Castro et al 1991, Jacques 1992, Gibson and Kernohan 1993, Basford 1993). The optical properties of tissues play an important and fundamental role in these applications because they directly determine how light behaves within the tissues. The optical properties of tissues are described by the absorption coefficient $\mu_{\mathrm{a}}\left(\mathrm{cm}^{-1}\right)$, scattering coefficient $\mu_{\mathrm{s}}\left(\mathrm{cm}^{-1}\right)$, and anisotropy factor $g$, which is the average cosine of the deflection angle after a single scattering event. One of the derived parameters is the total interaction coefficient $\mu_{\mathrm{t}}$, defined as $\mu_{\mathrm{a}}+\mu_{\mathrm{s}}$. Among the many methods of measuring these optical properties is the collimated light transmission method that measures total interaction coefficients (Cheong et al 1990, Flock et al 1992). This method is simple and involves minimal instrumentation. This note derives the error expression of this technique so that the optical properties can be measured with a controlled error.

First, an introduction will explain how the collimated transmission method works for a slab of tissue or a slab of turbid medium. Second, the number of transmitted photons with different numbers of scattering events assuming an ideal medium, $g=1$, will be derived. Third, using the set-up geometry and the scattering phase function of the medium, the collection efficiency of the detector for singly scattered light will be derived. Fourth, the error of measuring total interaction coefficients will be formulated based on the results of the second and third steps. Fifth, the error expression will be confirmed with accurate Monte Carlo simulation results.

\section{Collimated transmission method}

In a collimated transmission measurement (Cheong et al 1990, Flock et al 1992), a laser beam is directed perpendicularly to the surface of the sample slab. The collimated transmission is measured by rejecting the scattered light with the two apertures (figure 1). 
The signal $I_{0}$ is measured with a clear medium of a matching refractive index before the signal $I_{\mathrm{s}}$ is measured with the sample, where the sample can be a piece of tissue or a tissue phantom (e.g. polystyrene spheres solution). Based on Beer's law, the total interaction coefficient of the sample is computed by

$$
\mu_{\mathrm{t}}=\ln \left(I_{0} / I_{s}\right) / d
$$

where $d$ is the thickness of the sample.

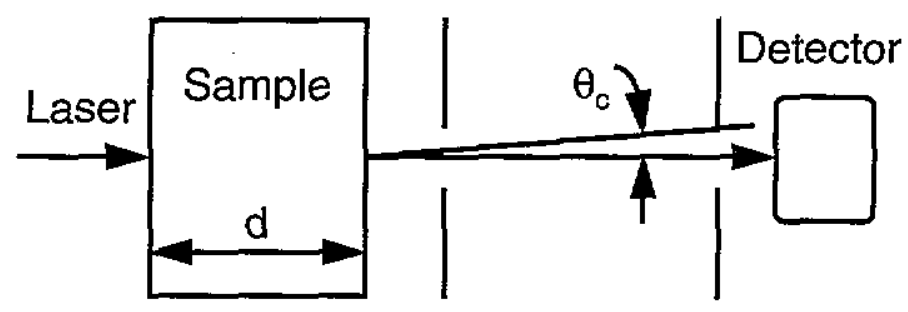

Figure 1. Schematic of the experimental set-up used for measuring total interaction coefficients with collimated transmission.

The validity of the measurement is based on the assumption that the detected scattered light is negligible compared with the detected unscattered light. Two factors affect this assumption: one is the collection angle $\theta_{\mathfrak{c}}$ (half angle) of the detector, and the other is the scattering optical depth $\mu_{s} d$ of the sample slab. The collection angle determines the percentage of scattered photons collected by the detector, which is called 'collection efficiency' in this article. The scattering optical depth determines the number of scattered photons compared with the unscattered photons.

\section{Transmission distribution}

Consider a sample slab of thickness $d$ with the following optical properties: matched index of refraction with the ambient medium, $\mu_{\mathrm{a}}, \mu_{\mathrm{s}}$, and $g=1$. If the total number of incident photons is $N_{\text {in }}$, then according to Beer's law the number of unscattered transmitted photons, $N_{0}$ is

$$
N_{0}=N_{\text {in }} \exp \left(-\mu_{\mathrm{t}} d\right) .
$$

Let the number of transmitted photons that have experienced $i$ scatterings be denoted by $N_{i}$, which is computed by

$$
N_{i}=N_{\text {in }} \exp \left(-\mu_{\mathrm{t}} d\right)\left(\mu_{\mathrm{s}} d\right)^{i} / i !
$$

where $i=0,1,2, \ldots$. Equation (3) is reduced to Poisson distribution if $\mu_{\mathrm{a}}=0$ and is a special case of the solution to the transport equation under small-angle approximation (Deepak et al 1982). Although the derivation can be modified from that of Poisson distribution, it is presented in the appendix for completeness. 


\section{Collection efficiency}

The unscattered light is assumed to be collimated and is completely collected by the detector. The singly scattered light has an angular distribution and is only partially collected, where the collection efficiency can be computed from the angular distribution and the collection angle of the detector.

The Henyey-Greenstein phase function (Henyey and Greenstein 1941, Jacques and Wang 1994), which is the probability density function of the cosine of the deflection angle $\theta$, is assumed for scatterings

$$
p(\cos \theta)=\left(1-g^{2}\right) /\left[2\left(1+g^{2}-2 g \cos \theta\right)^{3 / 2}\right]
$$

where $\theta$ is in the range $[0, \pi]$, and the integration of $p(\cos \theta)$ with $\cos \theta$ in the range $[-1,1]$ is unity.

For a sample slab in air with a refractive index $n$, the collection angle in air $\theta_{c}$ corresponds to a collection angle in sample $\theta_{c}^{\prime}$ that is computed by Snell's law, i.e. $\sin \left(\theta_{\mathrm{c}}\right)=n \sin \left(\theta_{\mathrm{c}}^{\prime}\right)$. Integrating (4) with $\cos \theta$ in the range [ $\left.\cos \theta_{\mathrm{c}}^{\prime}, 1\right]$, we obtain the fraction of single scattered light that is collected by the detector

$$
\chi=\left[1-(1-g) / \sqrt{1+g^{2}-2 g \cos \theta_{c}^{\prime}}\right](1+g) /(2 g)
$$

which is rather complicated but can be simplified to

$$
\chi \approx \theta_{\mathrm{c}}^{\prime 2} /\left[2(1-g)^{2}\right]=\theta_{\mathrm{c}}^{2} /\left[2 n^{2}(1-g)^{2}\right] .
$$

when $1-g \ll 1$, i.e. $g \rightarrow 1$, and $\theta_{\mathrm{c}}^{\prime} \ll 1-g$.

\section{Error expression}

When the scattering optical depth $\mu_{\mathrm{s}} d$ is of the order of 1 , we can consider the singly scattered light as noise and ignore multiply scattered light in order to estimate the detection error. For $g \rightarrow 1$, the number of singly scattered photons can be approximated by $N_{1}$ in (3). Therefore, the measurement error is approximately

$$
\epsilon=\chi N_{\mathrm{I}} / N_{0}
$$

where $\chi N_{1}$ is the number of collected singly scattered photons. Although the specular reflections on both slab surfaces are not considered in estimating the collimated and collected singly scattered photons by $N_{0}$ and $\chi N_{1}$, they experience approximately the same specular reflection. Therefore, the constant correction factors for the numerator and denominator in (7) caused by the specular reflection cancel out. Substituting the expressions for $N_{0}$ and $N_{1}$ based on (3) into (7), we obtain

$$
\epsilon=\chi \mu_{s} d \text {. }
$$

Substituting (6) into (8) and solving for $d$, we obtain

$$
d=2 \epsilon n^{2}(1-g)^{2} /\left(\theta_{\mathfrak{c}}^{2} \mu_{\mathrm{s}}\right)
$$

which is valid when $1-g \ll 1$ and $\theta_{c}^{\prime} \ll 1-g$. Equation (9) means that the sample thickness must be limited according to the range of the optical properties of the turbid medium and the collection angle of the detector in order to achieve a given accuracy. If one wishes to achieve $\epsilon \leqslant 1 \%$, where $n=1.37, g=0.99, \theta_{\mathrm{c}}=1 \mathrm{mrad}$ and $\mu_{\mathrm{s}}$ is approximately $100 \mathrm{~cm}^{-1}$, then $d$ should be $\leqslant 0.037 \mathrm{~cm}$. 

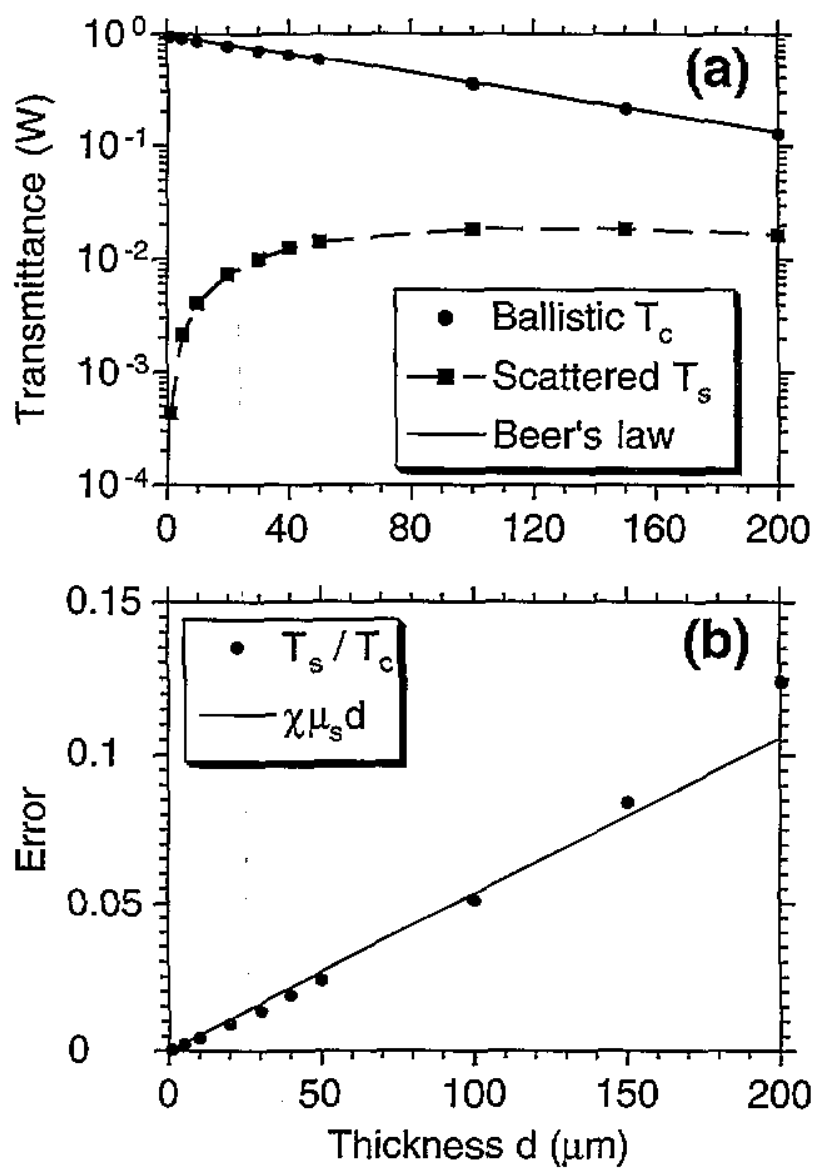

Figure 2. (a) Monte Carlo simulated unscattered collimated transmission, $T_{\mathrm{c}}$, and scattered transmission falling within the collection angle, $T_{s}$, through a sampie slab as a function of tissue thickness. The power of the laser beam is $1 \mathrm{~W}$, and the collection angle $\theta_{\mathrm{c}}$ is $2.5^{\circ}$. The optical properties of the medium are refractive index $=1.37, \mu_{\mathrm{a}}=0.1 \mathrm{~cm}^{-1}, \mu_{\mathrm{s}}=100 \mathrm{~cm}^{-1}$, and $g=0.9$. The solid line crossing the solid circles is created using Beer's law, i.e. $\left(1-R_{\mathrm{sp}}\right)^{2} \exp \left(-\mu_{1} d\right)$, where $R_{\mathrm{sp}}$ is the specular reflection equal to $2.4 \%$. (b) The error caused by scattered light is computed by $T_{\mathrm{s}} / T_{\mathrm{c}}$, which is closely approximated by (8), as shown by the solid line.

\section{Monte Carlo verification}

The validity of (8) was tested by Monte Carlo simulations (Wilson and Adam 1983, Prahl et al 1989, Wang and Jacques 1992, Jacques and Wang 1994) of the experiment. The unscattered collimated transmissions $T_{c}$ and the scattered transmission falling within the collection angle $T_{s}$ were computed for a laser beam perpendicularly incident to a tissue slab (figure 2(a)), because the scattering optical depth of the turbid medium in this simulation is limited to $2\left(100 \mathrm{~cm}^{-1} \times 0.02 \mathrm{~cm}\right)$ and the scattering is highly forward-directed, the radial spread of transmitted light is neglected in determining whether the light is collected by the detector. In other words, as long as the exit angle of the transmitted light with respect to the optical axis is less than the collection angle, the light is considered to be collected by the 
detector. The ratio $T_{\mathrm{s}} / T_{\mathrm{c}}$ represents the error and is closely approximated by (8) when $d$ is small, but error is underestimated by $(8)$ when $d$ is large (figure $2(b)$ ). The underestimation is due to the multiply scattered light. Therefore, (8) is only valid when $\mu_{s} d \leqslant 1$, which should be true in practice to achieve a good accuracy.

\section{Summary}

We have derived a practical error expression for measuring the total interaction coefficient with the collimated transmission (equation (8)). Although the derivation was based on the simple analytical expressions of photon-transmission distribution, the validity of the error expression was verified using accurate Monte Carlo simulations.

\section{Acknowledgments}

We thank Linda Eppich for proofreading the manuscript. This research was supported in part by the Whitaker Foundation, the Office of Naval Research N00014-91-J-1354, the Air Force Office of Scientific Research F49620-93-1-0298DEF, the National Institutes of Health R29-HL45045, and the Department of Energy DE-FG05-91ER61226.

\section{Appendix}

This appendix proves equation (3). An infinitely narrow photon beam is incident to the tissue along the $z$-axis, where the $z$-axis is perpendicular to the tissue surface and originates on the tissue surface. Let us define $P_{i}\left\{z_{1}, z_{2}\right\}$ as the probability of a photon travelling from $z_{1}$ and arriving at $z_{2}$ in the ideal tissue $(g=1)$ with $i$ scatterings, where $P_{i}\left\{z_{1}, z_{2}\right\}$ is only. a function of $z_{2}-z_{1}$. Equation (3) is equivalent to

$$
P_{i}\{0, d\}=\exp \left(-\mu_{\mathrm{t}} d\right)\left(\mu_{\mathrm{s}} d\right)^{i} / i !
$$

Induction will be used to prove (A1). First, (A1) is valid for $i=0$ because of (2). Second, we will prove if (A1) is true for $i=j$, then (A1) is true for $i=j+1$.

The probability of no scatterings between 0 and $z$ is $P_{0}\{0, z\}$. According to the definition of the scattering coefficient, the probability of a single scattering between $z$ and $z+\Delta z$ is

$$
P_{1}\{z, z+\Delta z\}=\mu_{s} \Delta z
$$

ignoring the probability of two or more scatterings, where $\Delta z$ is a small finite increment of $z$. The probability of $j$ scatterings between $z+\Delta z$ and $d$ is $P_{j}\{z+\Delta z, d\}$ which can be expanded to a zero-order term and a first-order error term, i.e.

$$
P_{j}\{z+\Delta z, d\}=P_{j}\{z, d\}+P_{j}^{\prime}\{z+k \Delta z, d\} \Delta z
$$

where $k$ is a constant between 0 and 1 , and $P_{j}^{\prime}\{z+k \Delta z, d\}$ is a first derivative with respect to $z$. Consequently, the probability that transmitted photons experience no scatterings between 0 and $z$, one scattering between $z$ and $z+\Delta z$, and $j$ scatterings between $z+\Delta z$ and $d$ is $P_{0}\{0, z\} P_{1}\{z, z+\Delta z\} P_{j}\{z+\Delta z, d\}$, which becomes

$$
P_{0}\{0, z\} P_{1}\{z, z+\Delta z\} P_{j}\{z+\Delta z, d\} \rightarrow \exp \left(-\mu_{\mathrm{t}} z\right) \mu_{\mathrm{s}} P_{j}\{z, d\} d z
$$


by letting $\Delta z \rightarrow 0$, replacing $\Delta z$ with $d z$, and ignoring the second-order infinitesimal term. Replacing $P_{j}\{z, d\}$ with (A1) for $i=j$ in which $d$ is replaced with $d-z$ and integrating (A4) over the full thickness of the tissue to cover all cases of $j+1$ scatterings, we obtain

$$
P_{j+1}\{0, d\}=\exp \left(-\mu_{i} d\right)\left(\mu_{\mathrm{s}} d\right)^{j+1} /(j+1) !
$$

which means (A1) is valid for $i=j+1$. Therefore (A1) is valid for all $i$ values based on this induction.

\section{References}

Basford J R 1993 Laser therapy: scientific basis and clinical role Orthopedics 16 541-7

Castro D J, Saxton R E, Lufkin R B, Haugland R P, Zwarun A A, Fetterman H R, Soudant J, Ward P Ff and Kangarloo H 1991 Future directions of laser phototherapy for diagnosis and treatment of malignancies: fantasy, fallacy, or reality? Laryngoscope 101 1-10

Cheong W F, Prahl S A and Welch A J 1990 A review of the optical properties of biological tissues IEEE J. Quantum Electron. 26 2166-85

Deepak A, Zardecki A, Farrukh U O and Box M A 1982 Multiple scattering effects of laser bearns traversing dense aerosols Atmospheric Aerosols: Their Formation, Optical Properties, and Effect ed A Deepak (Virginia: Spectrum) pp 185-225

Flock S T, Jacques S L, Wilson B C, Star W M and van Gemert M J 1992 Optical properties of Intralipid: a phartom medium for light propagation studies Laser Surg. Med. $12510-9$

Gibson K F and Kernohan W G 1993 Lasers in medicine-a review J. Med. Eng. Technol. 17 51-7

Henyey L G and Greenstein J L 1941. Diffuse radiation in the galaxy Astrophys. J. 93 70-83

Jacques S L 1992 Laser-tissue interactions: photochemical, photothermal, and photomechanical Lasers General Surg. 72 531-58

Jacques S L and Wang L-H 1994 Monte Carlo modeling of light transport in tissues Optical Thermal Response of Laser Irradiated Tissue ed A J Welch and M J C van Gemert (New York: Plenum) at press

Prahl S A, Keijzer M, Jacques S L and Welch A J 1989 A Monte Carlo model of light propagation in tissue Proc. Soc. Photo-Opt. Instrum. Eng. IS 5 102-11

Shapshay S M 1989 Laser technology in the diagnosis and treatment of head and neck cancer Semin. Surg. Oncol. $561-6$

Wang L-H and Jacques S L 1992 Monte Carlo Modeling of Light Transport in Multi-Layered Tissues in Standard $C$ The University of Texas M D Anderson Cancer Center, Houston, TX, USA

Wilson B C and Adam G 1983 A Monte Carlo model for the absorption and fux distributions of light in tissue Med. Phys. 10 824-30 